Research Article

\title{
Design of an S-Band Phased Array with Modified Dipoles
}

\author{
Meng Xiang, Yu Xiao $(\mathbb{D}$, Bin Xi, Yue Zhang, and Shiyou Xu \\ School of Electronics and Communication Engineering, Sun Yat-sen University, Shenzhen 518107, China \\ Correspondence should be addressed to Yu Xiao; xiaoy283@mail.sysu.edu.cn
}

Received 2 June 2021; Revised 5 October 2021; Accepted 25 October 2021; Published 29 November 2021

Academic Editor: Ahmed Toaha Mobashsher

Copyright $\odot 2021$ Meng Xiang et al. This is an open access article distributed under the Creative Commons Attribution License, which permits unrestricted use, distribution, and reproduction in any medium, provided the original work is properly cited.

A wideband, low cross-polarization, high-gain, and wide-angle scanning antenna array is presented in this paper. The antenna array contains 8 subarrays in the horizontal dimension, and each subarray contains 4 unit cells. A two-side printed dipole with an amendatory equivalent circuit model is adopted, and the metal vias are introduced in the element design to ameliorate the crosspolarization level. A radome, acting as the wide-angle impedance matching layer, is introduced to achieve wide-angle scanning. A prototype of a $4 \times 8$ array is fabricated and measured. The results show that the operating bandwidth of aperture efficiency (BWAE) above $60 \%$ is about $26.7 \%$ from $2.6 \mathrm{GHz}$ to $3.4 \mathrm{GHz}$. The measured scanning loss in the $H$-plane is $2.7 \mathrm{~dB}$ when scanning up to $60^{\circ}$ with active voltage standing wave ratio (VSWR) $<3$, and the gain can achieve $21 \mathrm{~dB}$ at $3 \mathrm{GHz}$ with a cross-polarization level below $-30 \mathrm{~dB}$ at all angles.

\section{Introduction}

Phased-array antennas are widely used in enhancing the gain, angular resolution, and antijamming capability of radar systems and modern communication systems [1]. However, the scanning performance of the phased array often suffers from an increasing scanning loss and crosspolarization when the main beam is steered off the broadside direction [2]. This is mainly caused by the radiation performance and scanning impedance of the array elements.

The printed dipole antenna is one of the most wellknown forms in phased-array systems, owing to its advantages such as lightweight, low cost, ease of fabrication, and suitability for integration with microwave integrated circuit modules [3]. However, the printed dipole antenna that is directly fed has the problem of impedance mismatch in practical applications, which results in a narrow bandwidth [4]. Generally, a balun is used to facilitate the impedance matching and expand the bandwidth; it can also achieve a balanced conversion. In [5], the printed dipole with an integrated balun can match the feed simply through an adjustment of the feed point. In [6], a systematic approach is presented to describe planar slot antennas embedded in generic stratified media, and the transform between the slot and transmission line is researched. In [7], a novel microstrip balun topology based on common-mode rejection is introduced to achieve a successful conversion from a single end to a differential mode. These research studies will help to analyze the printed dipole with an integrated balun.

Besides, the cross-polarization level is not good due to an asymmetric feeding and radiation structure. To achieve low cross-polarization of the antipodal dipole array, a tapered balun with the via, groove, and shorting line is designed in [8]. In [9], a wideband wide-scan phased array of connected dipoles has been designed and fabricated. By using a circular feed design and a common-mode suppression technology, the cross-polarization is reduced to below $-10 \mathrm{~dB}$ for an elevation angle up to $45^{\circ}$ in all azimuth planes. In [4], a double-layer dielectric plate is applied to balance the electric field distribution, and the cross-polarization level is reduced to below $-30 \mathrm{~dB}$. In [10], a low-profile shorted-patch antenna with enhanced bandwidth and the reduced $H$-plane cross-polarization is proposed, and the $H$-plane cross-polarization of the shorted-patch antenna is successfully decreased to below $-12.3 \mathrm{~dB}$ over the operating band.

In this paper, a modified element based on a balanced stripline structure is proposed, and its equivalent circuit 
model is introduced for a more precise design. Two sets of metalized vias are introduced to compensate for the asymmetry of the structure, and the cross-polarization of the unit cell can achieve less than $-58 \mathrm{~dB}$ in the range of $-10 \mathrm{~dB}$ of the main beam. In addition, a radome, acting as the wideangle impedance matching layer, is also introduced to enhance the wide-angle scanning performance. A prototype array has been fabricated and measured. The results show that the operating bandwidth of aperture efficiency above $60 \%$ is about $26.7 \%$ from $2.6 \mathrm{GHz}$ to $3.4 \mathrm{GHz}$. The measured scanning loss is only $2.7 \mathrm{~dB}$ when scanning up to $60^{\circ}$ with active VSWR $<3$, and the gain can achieve $21 \mathrm{~dB}$ at $3 \mathrm{GHz}$ with an extremely low cross-polarization level below $-30 \mathrm{~dB}$ at all angles.

This paper is organized as follows. Section 2 describes the antenna element and its principles. Section 3 presents the prototype of the designed array and the measured results. Section 4 concludes this work.

\section{Antenna Design}

The geometrical structure of the dipole unit cell and its exploded view are shown in Figures 1 and 2, respectively. The dipole is fabricated on two-layer substrates of F4BM220 with a dielectric constant of $\varepsilon_{\mathrm{r}}=2.2$. The thickness of each substrate is $0.765 \mathrm{~mm}$. Two substrates are bonded using a $0.1 \mathrm{~mm}$ bonding film. The proposed antenna element consists of three metal layers. Two T-shaped patches are printed on the upper and lower layers of the substrates. Two sets of metalized vias are introduced to connect the patches. The integrated balun consists of a coupled line on the middle layer and two slots on the upper and lower layers. The structural parameters are listed in Table 1.

To facilitate the optimization process, a modified equivalent circuit is introduced to analyze the antenna unit cells, as shown in Figure 3. The printed dipole with the balun can be considered as a simple dipole $\left(Z_{\mathrm{d}}\right)$ fed by a coupled line (divided into two parts, coupled line 1 and coupled line 2), and the shorted stub $\left(Z_{\mathrm{s}}\right)$ can be seen as a grounded stub. An additional matching line $\left(Z_{\mathrm{m}}\right)$ is inserted to improve impedance matching. In this model, the transmission line feeds both the right arm and the left arm of the dipole. So, the length of each coupled line in Figure 3 corresponds with the total length of the coupled line in Figure 1. Generally, an ideal transformer that has a turn ratio of $n$ is used to analyze the coupled structure as the equivalent circuit in [5]. However, it is complex for analysis. In this paper, two coupled lines are used instead of the ideal transformer in [5]. As a consequence, the coupling line has one-by-one correspondence with the physical structure, making parameter extraction more straightforward. This is conducive to understanding the principle of the antenna and optimizing its performance.

According to the above description, two coupling lines form a four-port coupling network (the fourth port is grounded). The input port is at port $\mathrm{A}$, and the output port is at port $\mathrm{B}$. The fourport $S$-parameters of the two asymmetric coupled lines, which have the electrical length of $\theta$, are given by [11]

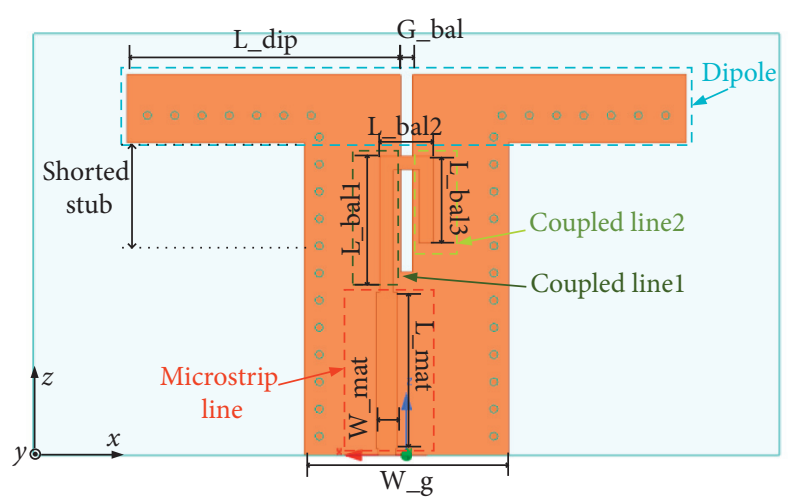

FIgURE 1: Structure of the dipole unit cell.

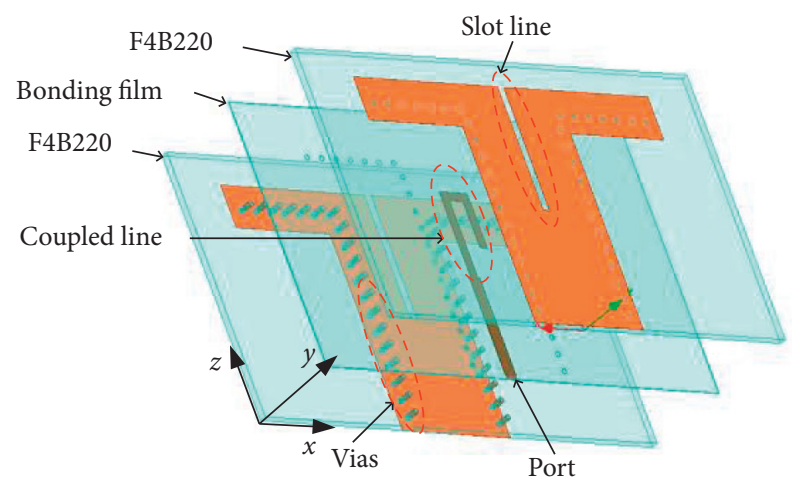

FIgURE 2: Exploded view of the dipole unit cell.

Table 1: Parameters of the dipole unit cell.

\begin{tabular}{lccc}
\hline Parameters & Value $(\mathrm{mm})$ & Parameters & Value $(\mathrm{mm})$ \\
\hline W_dip & 5.0 & L_bal1 & 10.0 \\
G_bal & 0.9 & L_bal2 & 3.9 \\
L_mat & 11.5 & L_bal3 & 6.4 \\
W_mat & 1.48 & L_g & 23.0 \\
W_g & 15.0 & & \\
\hline
\end{tabular}

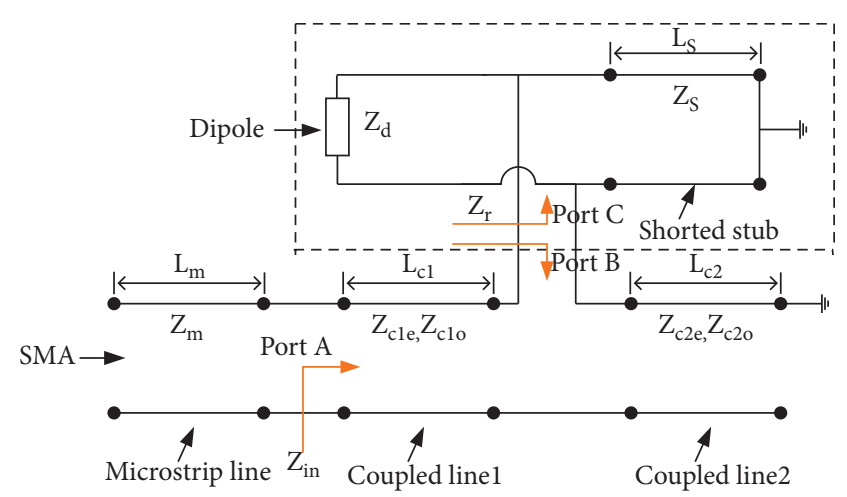

Figure 3: Equivalent circuit of the printed dipole with the balun. 


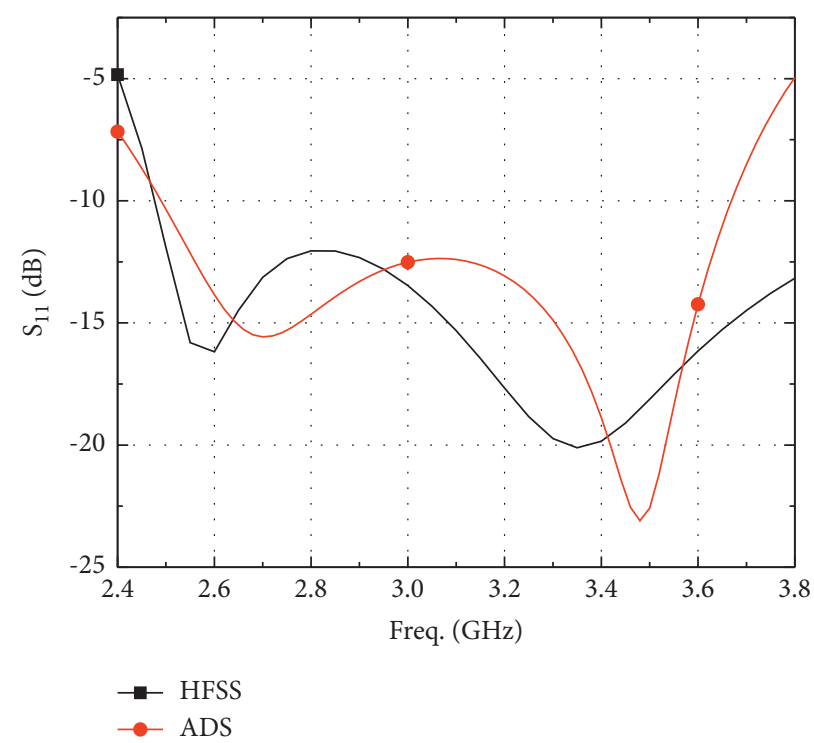

FIgURE 4: The simulated results of the equivalent circuit by ADS and full-wave calculation by HFSS.

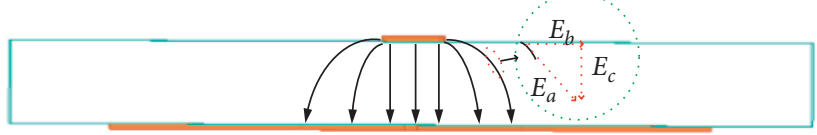

(a)

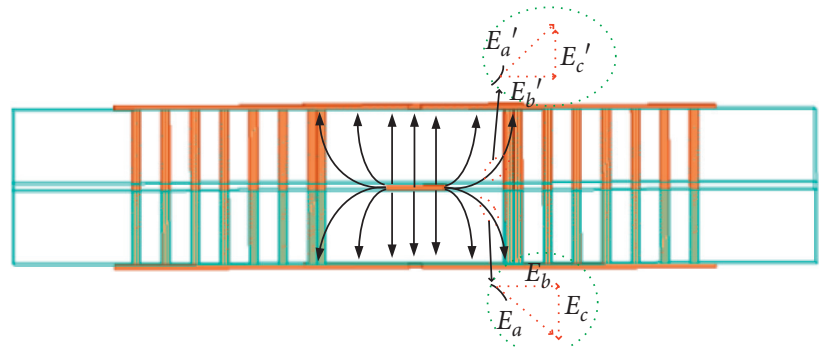

(b)

Figure 5: Electric field distribution of the antenna on the top view. (a) Microstrip structure. (b) Stripline structure.

$$
[S]_{\text {coupler }}=\left[\begin{array}{cccc}
0 & \frac{x}{z} & \frac{y}{z} & 0 \\
\frac{x}{2} & 0 & 0 & \frac{y}{z} \\
\frac{y}{z} & 0 & 0 & \frac{x}{z} \\
0 & \frac{y}{z} & \frac{x}{z} & 0
\end{array}\right],
$$

where

$$
\left\{\begin{array}{l}
x=\sqrt{1-k^{2}}, \\
y=j k \sin \theta, \\
z=\sqrt{1-k^{2}} \cos \theta+j \sin \theta,
\end{array}\right.
$$

and $k$ is the coupling coefficient.

Then, looking from port $\mathrm{C}$ in Figure 3, including the radiation impedance of the antenna and the short-circuit stub equivalent by the slot, their combined impedance $Z_{\mathrm{r}}$ can be expressed as

$$
Z_{r}=\frac{j Z_{d} Z_{s} \tan \beta_{s} l_{s}}{Z_{d}+j Z_{s} \tan \beta_{s} l_{s}},
$$

where $Z_{\mathrm{d}}$ is the dipole's resonant resistance, $Z_{\mathrm{s}}$ is the equivalent and simplified impedance of the short-circuited stub, $\beta_{\mathrm{s}}$ is the phase constant, and $l_{\mathrm{s}}$ is the length of the gap.

According to the above theoretical derivation, we can get the initial size based on the matching theory between the three-segment parts. The comparison of the simulation results between the equivalent circuit by ADS and the fullwave calculation by Ansys HFSS is shown in Figure 4. Good agreement is observed. It proves the effectiveness of the equivalent circuit.

In the design of the printed dipole antenna, the crosspolarization is an index that should pay much attention to it. Generally, the cross-polarization level is not good. In [5], the cross-polarization level is only below $-20 \mathrm{~dB}$. In [4], a double-layer dielectric plate is applied to balance the electric field distribution, and the cross-polarization level is reduced 


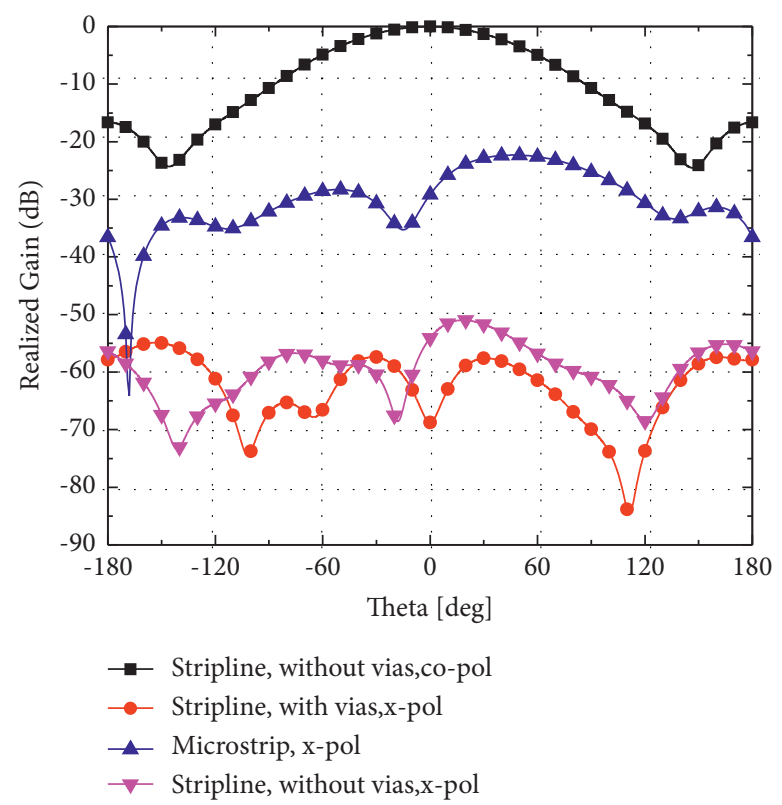

Figure 6: Comparison of the pattern ( $H$-plane) and cross-polarization of different structures.

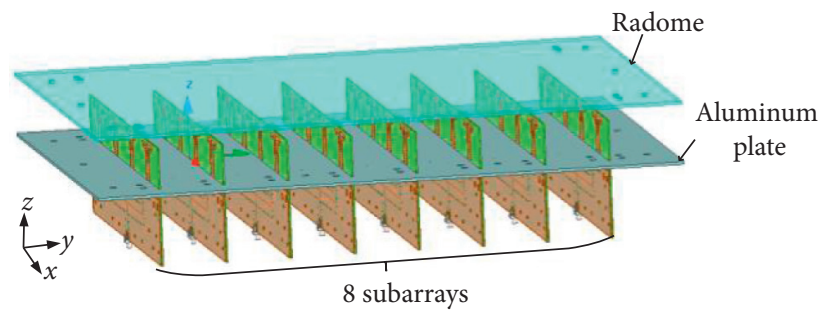

(a)

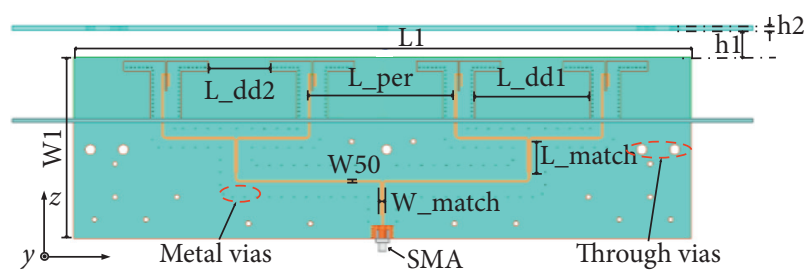

(b)

FIGURE 7: Geometry of the proposed antenna. (a) Antenna array. (b) Antenna subarray.

TABle 2: Parameters of the antenna subarray.

\begin{tabular}{lccc}
\hline Parameters & Value $(\mathrm{mm})$ & Parameters & Value $(\mathrm{mm})$ \\
\hline L1 & 300 & L-per & 71.2 \\
W1 & 84 & W_50 & 1.2 \\
L_dd1 & 56.2 & W_match & 2 \\
L_dd2 & 30.2 & L_match & 16 \\
\hline
\end{tabular}

to below $-30 \mathrm{~dB}$. The field distribution of the traditional single-side printed dipole antenna and the stripline printed dipole antenna is simply illustrated in Figure 5. It can be seen that, at the edge of the microstrip feed line in Figure 5(a), the tangential electric field $\left(E_{\mathrm{a}}\right)$ can be decomposed into a horizontal electric field $\left(E_{\mathrm{b}}\right)$ and a vertical electric field $\left(E_{\mathrm{c}}\right)$. $E_{\mathrm{b}}$ will be counteracted due to symmetry, but $E_{\mathrm{c}}$ will still exist. For the stripline in Figure $5(\mathrm{~b}), E_{\mathrm{b}}$ and $E_{\mathrm{c}}$ will be counteracted together because of symmetry. When they are used to feed the antenna, the stripline will have lower crosspolarization. In Figure 6, it can be seen that the cross-polarization of the microstrip printed dipole can barely reach $-20 \mathrm{~dB}$, while the stripline dipole has a cross-polarization of lower than $-50 \mathrm{~dB}$, but it is asymmetric about $0^{\circ}$ at all angles.

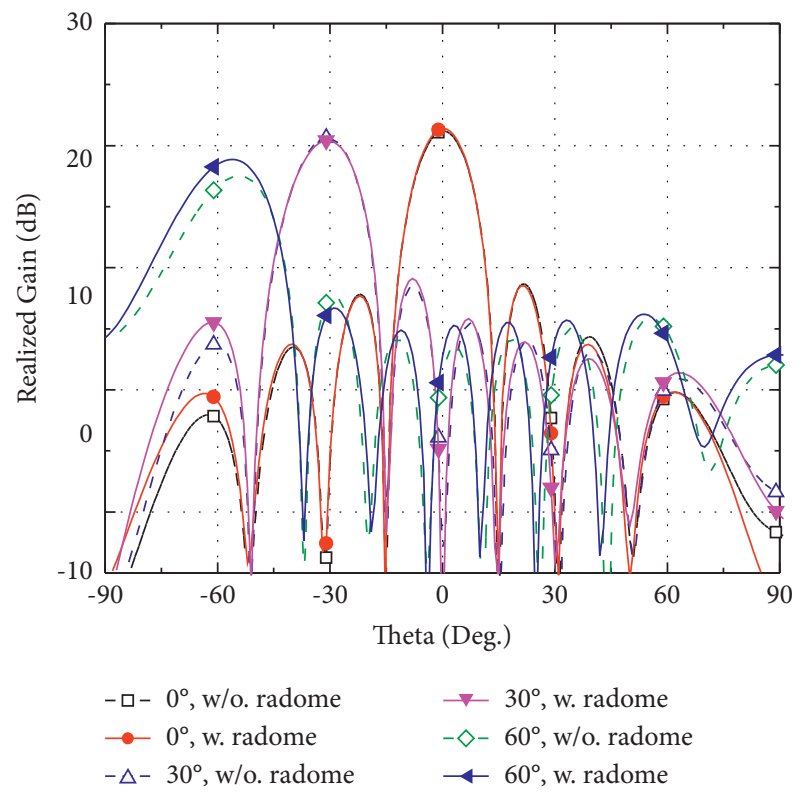

FIgURE 8: Comparison of simulation results ( $H$-plane) with and without the radome. 
TABLE 3: Comparison of the specific values in Figure 8.

\begin{tabular}{lccccc}
\hline Simulation & \multicolumn{3}{c}{ Without the radome $(3 \mathrm{GHz})$} & \multicolumn{2}{c}{ With the radome $(3 \mathrm{GHz})$} \\
\hline Direction & $0^{\circ}$ & $-30^{\circ}$ & $-60^{\circ}$ & $0^{\circ}$ & $-30^{\circ}$ \\
Gain (dB) & 21.2 & 20.7 & 17.5 & 21.4 & $-60^{\circ}$ \\
HPBW (deg) & 13 & 14.8 & 19.5 & 13 & 18.9 \\
SLL (dB) & 12.6 & 12 & 10.5 & 12.9 & 15 \\
\hline
\end{tabular}

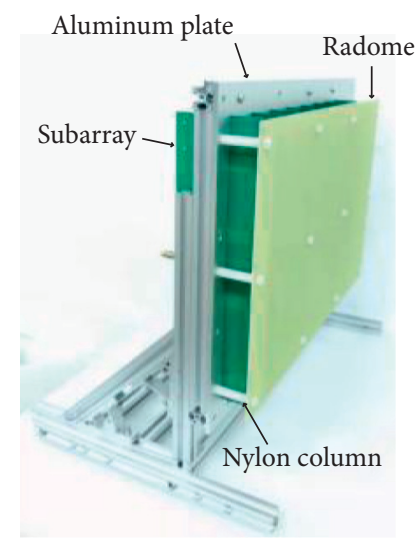

(a)

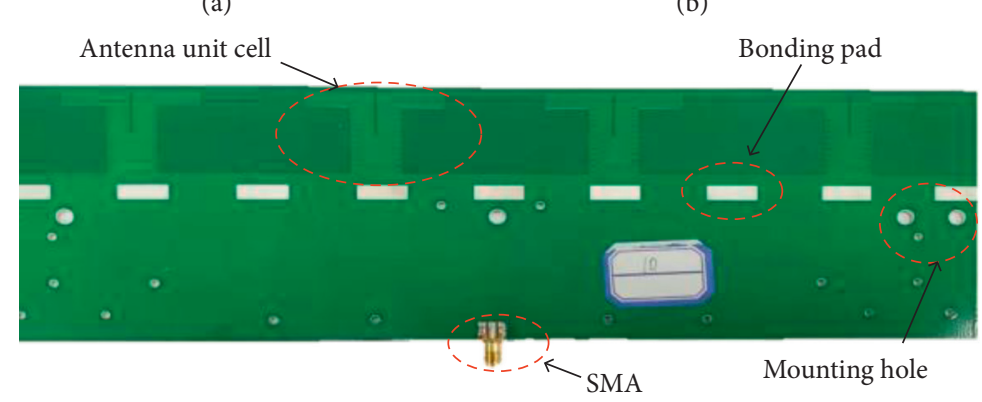

(c)

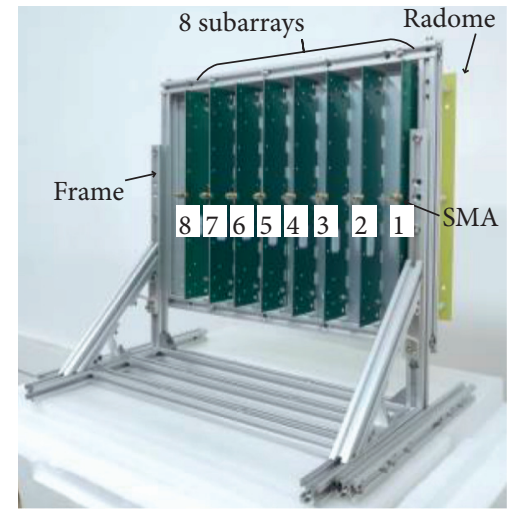

(b)

Figure 9: Photographs of the fabricated array containing the frame. (a) Front view. (b) Back view. (c) Antenna subarray.

The reason is that when the two layers are laminated, the presence of the bonding film will cause a slight asymmetry on both sides of the stripline.

To further optimize the cross-polarization performance, two sets of metalized vias are also introduced. It is clear that when adding the metalized vias, the cross-polarization curve is more symmetrical about $0^{\circ}$. In the range of $-10 \mathrm{~dB}$ of the main beam, the maximum value of cross-polarization decreases from $-51 \mathrm{~dB}$ to $-58 \mathrm{~dB}$. The introduction of metalized vias makes the two layers of radiation patches fully conductive, which has a good compensation effect. According to Figure 5(b), when there is a slight difference between $E_{\mathrm{c}}$ and $E_{c}^{\prime}$, they can be completely counteracted due to the compensation effect of the metal vias. It should also be pointed out that the maximum gain is not $0^{\circ}$ but offset by $0.4^{\circ}$ without the metal vias in Figure 6. However, it is not obvious in this array due to a wide beam, but this may be of importance for some high-gain and narrow beam arrays.

The array in this paper consisted of 8 subarrays and a radome, shown in Figure 7. Each subarray contains 4 antenna unit cells which are equally arranged in the $E$-plane ( $x$-direction) with a distance of about $0.7 \lambda_{0}$, and a $1 \times 4$ power divider is used to feed them, where $\lambda_{0}$ is the free-space wavelength at $3 \mathrm{GHz}$. Then, the eight subarrays are stacked in the $H$-plane ( $y$-direction) with a distance of about $0.5 \lambda_{0}$. Table 2 gives some key parameters of the antenna subarray.

Considering a radome is always needed for the antenna when operating outdoors, a substrate of FR- 4 with a dielectric constant of $\varepsilon_{\mathrm{r}}=4.2$ is adopted as the radome. It also plays the role of the impedance matching layer, selected the thickness of $h_{2}=2 \mathrm{~mm}$. In Figure 8, the improvement of the optimized radome on the scanning dimension ( $H$-plane) is displayed. It can be seen that the scanning performance has been improved at large scanning angles. A detailed comparison between the results of the array with and without the radome is given in Table 3 . The realized gain of the $60^{\circ}$ scanning angle increases from $17.5 \mathrm{~dB}$ to $18.9 \mathrm{~dB}$; that is, the loss at $60^{\circ}$ decreases from the original $3.7 \mathrm{~dB}$ to $2.5 \mathrm{~dB}$. The purpose of $60^{\circ}$ wide-angle scanning in the $H$-plane is achieved.

\section{Results of the Fabricated Array}

Based on the proposed unit cell, a prototype with $4 \times 8$ elements, as shown in Figure 9, is designed and fabricated to 


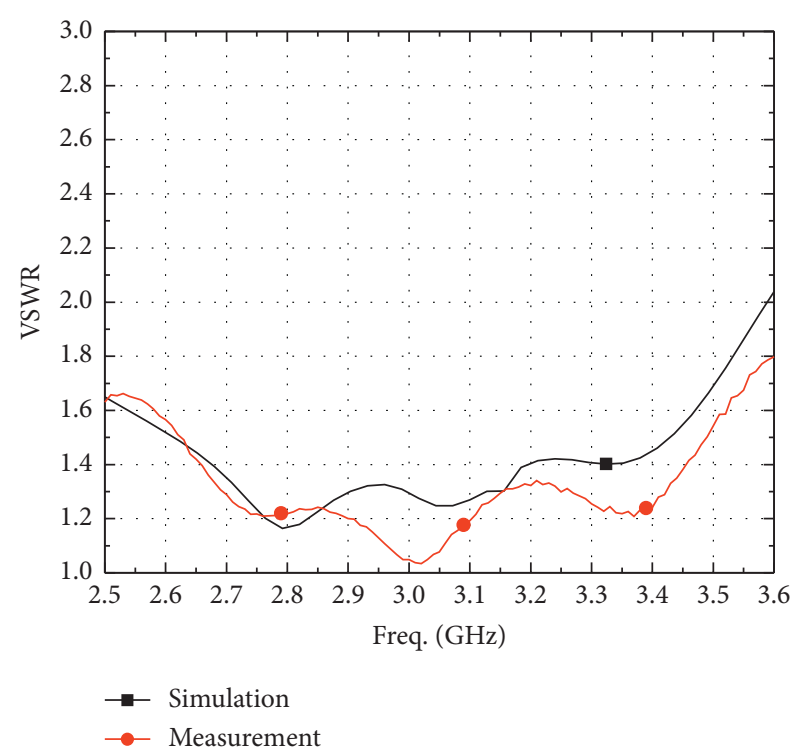

(a)

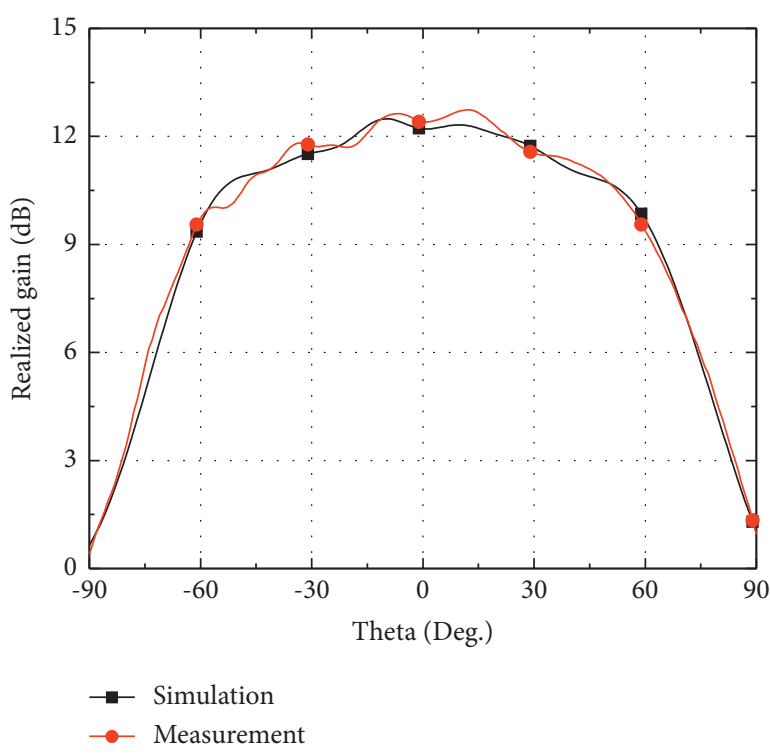

(b)

FIgURE 10: The results of the simulated and measured subarray. (a) VSWR. (b) Radiation patterns (3 GHz).

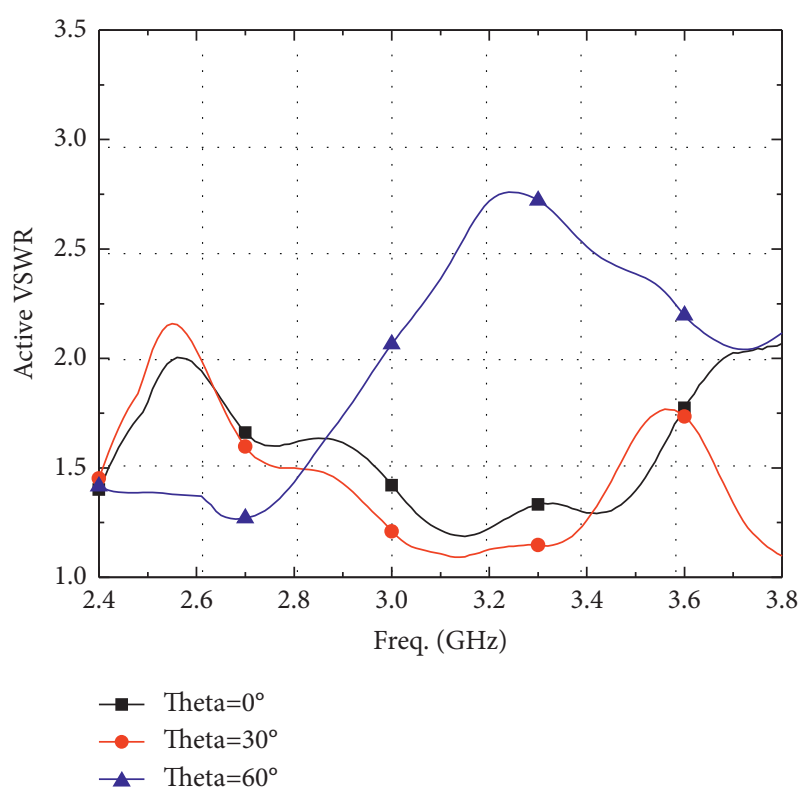

Figure 11: Active VSWR under different scanning angles along the $H$-plane (no. 5).

experimentally verify the cross-polarization and the beam scanning performance. Plastic columns are inserted into the array to hold the radome. To facilitate installation and measurement, a frame is designed using aluminum profiles.

The reflection coefficient and radiation pattern of the subarray are shown in Figure 10. During the measurement, the fifth subarray was excited, and the other subarrays were terminated with $50 \Omega$ loads. It can be seen that the measured VSWR and radiation pattern coincide with the simulated one very well.

The active VSWR of the phased array can be obtained from the coupling coefficients between the elements. When

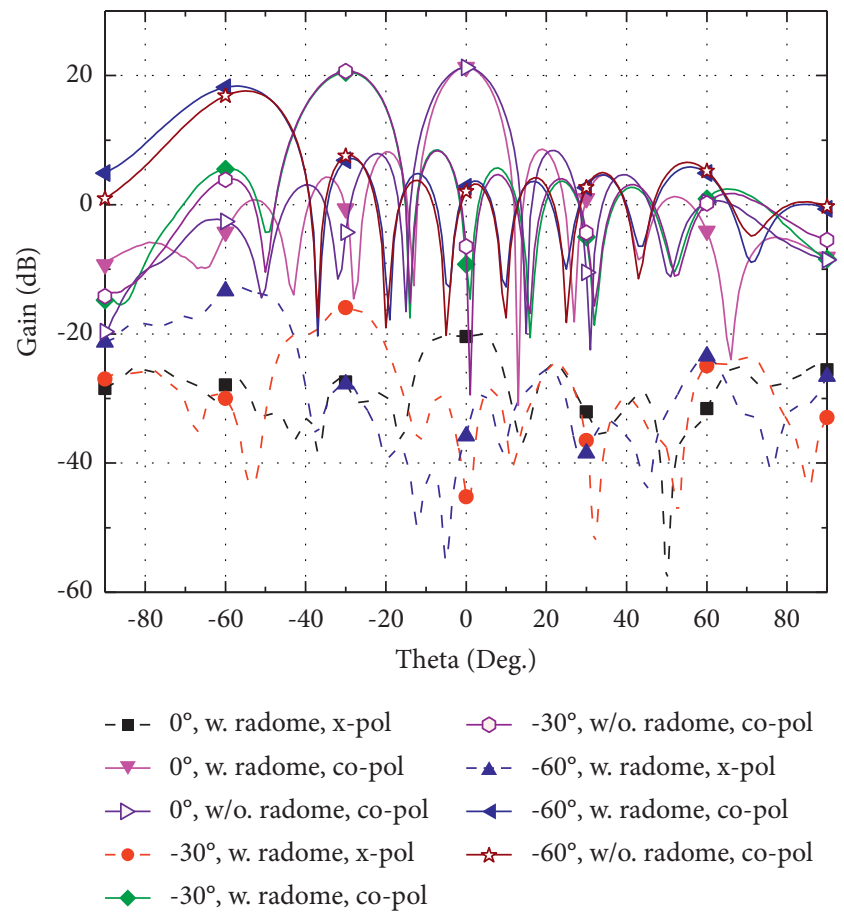

FIGURE 12: The synthesized results of beam scanning ( $H$-plane) at $3 \mathrm{GHz}$.

the elements $i$ and $n$ need to be measured, the other elements are matched. The active reflection coefficient for element $i$ can be estimated using $[12,13]$

$$
\Gamma_{\mathrm{a}}\left(\theta_{0}, \varphi_{0}\right)=\sum_{n=1}^{N} S_{n i}\left|a_{n}\right| e^{-j k_{0}\left(x d_{x} \sin \theta_{0} \cos \varphi_{0}+y d_{y} \sin \theta_{0} \sin \varphi_{0}\right),}
$$




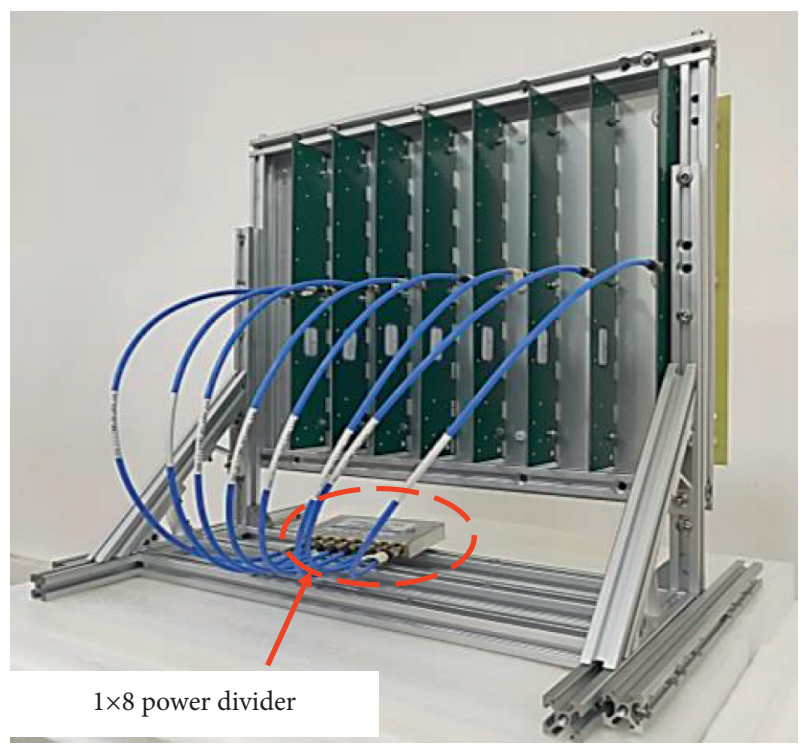

FIGURE 13: The measured scheme of the array with a power divider.

where $S_{\mathrm{ni}}$ is the coupling coefficient between elements, $\left(\theta_{0}\right.$, $\left.\varphi_{0}\right)$ is the scanning direction, $\left|a_{n}\right|$ is the magnitude of the excitation of the radiating element, $N$ is the total number of elements, $k_{\mathrm{o}}$ is the wavenumber of free space, $x$ and $y$ are the element numbers, and $d_{x}$ and $d_{y}$ are the element spacing along the $x$ - and $y$-directions, respectively. It can be seen from Figure 11 that active VSWR gradually deteriorates as the scanning angle increases, but within the BWAE range, active VSWR is below 3 .

The radiation patterns of the array can be synthesized from the measured results of each array element, which is calculated by [14]

$$
P_{\text {array }}=\sum_{n=1}^{N} A_{n} P_{n}(\theta, 0) e^{j\left[(n-1) k d \sin (\theta)-\phi_{n}\right]},
$$

where $d$ is the spacing between the adjacent elements and $A_{n}$ and $\phi_{n}$ are the input amplitude and phase of the $n$th port, respectively. Each measured result in (5) is obtained by exciting one port of the array, while all the other ports are terminated with matched loads [15]. Here, a uniform excitation with a constant amplitude and linear phase change is used for all feeding ports during the measurements.

The synthesized radiation patterns and cross-polarization at $3 \mathrm{GHz}$ are shown in Figure 12. It can be seen that the gain attenuation of the antenna is $2.7 \mathrm{~dB}$ when scanning to $60^{\circ}$. The half-power angle of the broadside main beam is approximately $13^{\circ}$. At the same time, it can be found that the cross-polarization becomes worse as the scanning angle increases, but it can still be maintained below $-30 \mathrm{~dB}$ when scanning to $60^{\circ}$.

To further verify the array's performance, a $1 \times 8$ Wilkinson power divider (as marked with a dotted line in Figure 13) is used to feed the 8 subarrays synchronously with almost equal phase and amplitude to get a broadside direction radiation. As shown in Figure 14, the bandwidth of aperture efficiency above $60 \%$ is from $2.6 \mathrm{GHz}$ to $3.4 \mathrm{GHz}$. The comparison between measured and synthesis gains is

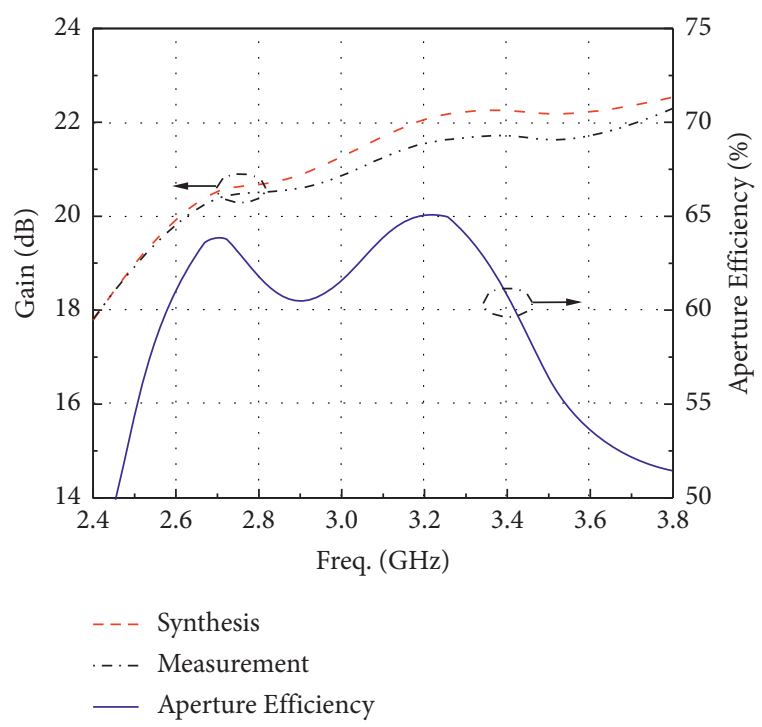

FIGURE 14: The synthesis aperture efficiency and the comparison of measured and synthesis gains.

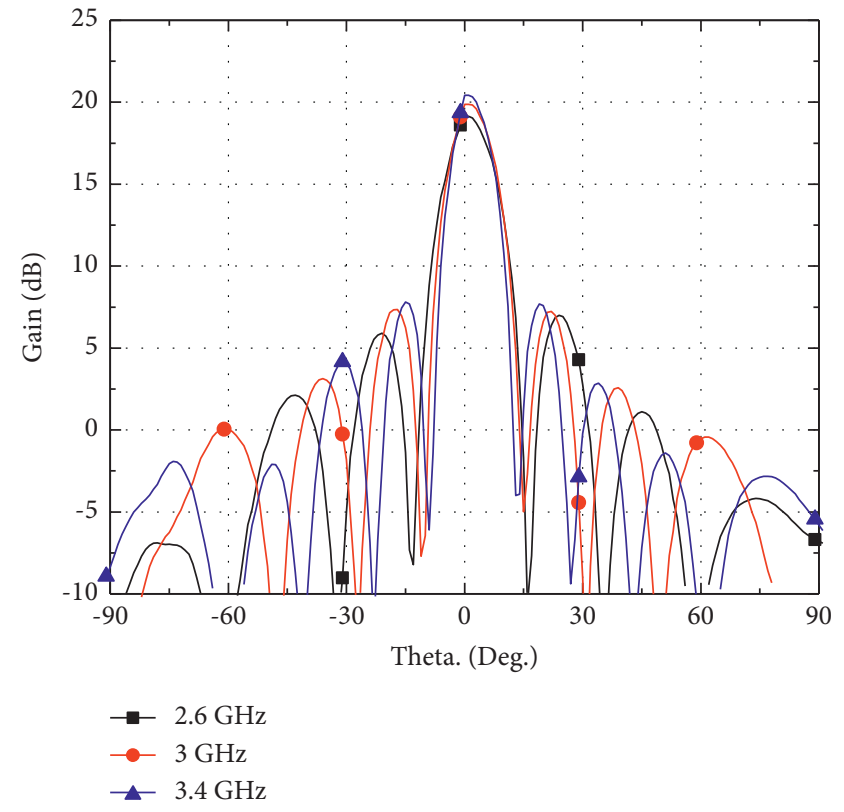

FIgURE 15: Measured radiation pattern ( $H$-plane) of the antenna array.

TABLE 4: Comparison of measured results and synthesis results $\left(0^{\circ}\right)$.

\begin{tabular}{lcccccc}
\hline$f(\mathrm{GHz})$ & \multicolumn{2}{c}{2.6} & \multicolumn{2}{c}{3} & \multicolumn{2}{c}{3.4} \\
WAIM & Mea. & Syn. & Mea. & Syn. & Mea. & Syn. \\
\hline Gain (dB) & 20.2 & 20.4 & 20.9 & 21.5 & 21.4 & 22 \\
HPBW (deg) & 13.5 & 13 & 12.8 & 12.5 & 12.5 & 12 \\
SLL (dB) & 12.7 & 13.3 & 12.2 & 12.7 & 12.4 & 12.5 \\
\hline
\end{tabular}

also shown in Figure 14. It can be seen that the measured result is slightly lower than the synthesized result within $0.5 \mathrm{~dB}$. The measured radiation patterns are plotted in Figure 15. The key parameters are also listed in Table 4. It can 
be seen that the measured realized gain, beamwidth, and sidelobe level (SLL) are very close to the synthesized results. The measured realized gain at $2.6 \mathrm{GHz}, 3 \mathrm{GHz}$, and $3.4 \mathrm{GHz}$ is $20.2 \mathrm{~dB}, 20.9 \mathrm{~dB}$, and $21.4 \mathrm{~dB}$, respectively, and the measured beamwidth and sidelobe level are about $12.8^{\circ}$ and $12.2 \mathrm{~dB}$, respectively. In general, it proves that the synthesis method and the synthesized results in Figure 12 are reliable.

\section{Conclusion}

In this paper, a phased antenna array with WAIM is designed to achieve wideband, low cross-polarization, highgain, and wide-angle scanning. An amendatory equivalent circuit model of the printed dipole is introduced for a more precise design. Compared with the previous work, the proposed circuit model has one-by-one correspondence with the physical structure, making parameter extraction more straightforward. Then, a modified element based on a balanced stripline structure is proposed, and two sets of metalized vias are also introduced to compensate for the asymmetry of the structure. As a result, in the range of $-10 \mathrm{~dB}$ of the main beam, the maximum value of crosspolarization decreases from $-51 \mathrm{~dB}$ to $-58 \mathrm{~dB}$. A substrate radome, acting as the wide-angle impedance matching layer, is also introduced to enhance the wide-angle scanning performance. To verify it, a prototype array has been fabricated and measured. The results show that the operating bandwidth of aperture efficiency above $60 \%$ is about $26.7 \%$ from $2.6 \mathrm{GHz}$ to $3.4 \mathrm{GHz}$. The measured scanning loss is only $2.7 \mathrm{~dB}$ when scanning up to $60^{\circ}$ with active $\operatorname{VSWR}<3$, and the gain can achieve $21 \mathrm{~dB}$ with a low cross-polarization level below $-30 \mathrm{~dB}$ at all angles at $3 \mathrm{GHz}$.

\section{Data Availability}

The data used to support the findings of this study are included within the article.

\section{Conflicts of Interest}

The authors declare that they have no conflicts of interest.

\section{Acknowledgments}

This work was supported in part by the Natural Science Foundation of China (Grant no. 62001522).

\section{References}

[1] M. I. Skolnik, Introduction to Radar Systems, McGraw-Hill, New York, NY, USA, 3rd ed. edition, 2001.

[2] G. Yang, J. Li, S. G. Zhou, and Y. Qi, "A wide-angle E-plane scanning linear array antenna with wide beam elements," IEEE Antennas and Wireless Propagation Letters, vol. 16, pp. 2923-2926, 2017.

[3] K. Fujimoto and J. R. James, Mobile Antenna System Handbook, Artech House, Boston, MA, USA, 1994.

[4] Z. Zhou, S. Yang, and Z. Nie, "A novel broadband printed dipole antenna with low cross-polarization," IEEE Transactions on Antennas and Propagation, vol. 55, no. 11, pp. 3091-3093, 2007.
[5] R. Li, T. Wu, B. Pan, K. Lim, J. Laskar, and M. M. Tentzeris, "Equivalent-circuit analysis of a broadband printed dipole with adjusted integrated balun and an array for base station applications," IEEE Transactions on Antennas and Propagation, vol. 57, no. 7, pp. 2180-2184, 2009.

[6] R. M. Van Schelven, D. Cavallo, and A. Neto, "Equivalent circuit models of finite slot antennas," IEEE Transactions on Antennas and Propagation, vol. 67, no. 7, pp. 4367-4376, 2019.

[7] M. Kim, "Broadband coupled-line balun on a defected ground structure," Microwave and Optical Technology Letters, vol. 54, no. 11, pp. 2575-2577, 2012.

[8] B. Wang, S. Yang, Y. Chen, S. Qu, and J. Hu, "Low crosspolarization ultrawideband tightly coupled balanced antipodal dipole array," IEEE Transactions on Antennas and Propagation, vol. 68, no. 6, pp. 4479-4488, 2020.

[9] D. Cavallo, A. Neto, G. Gerini, A. Micco, and V. Galdi, “A 3to $5-\mathrm{GHz}$ wideband array of connected dipoles with low cross polarization and wide-scan capability," IEEE Transactions on Antennas and Propagation, vol. 61, no. 3, pp. 1148-1154, 2013.

[10] N.-W. Liu, L. Zhu, G. Fu, and Y. Liu, "A low profile shortedpatch antenna with enhanced bandwidth and reduced $\mathrm{H}$-plane cross-polarization," IEEE Transactions on Antennas and Propagation, vol. 66, no. 10, pp. 5602-5607, 2018.

[11] C.-S. Lin, P.-S. Wu, M.-C. Yeh et al., "Analysis of multiconductor coupled-line marchand baluns for miniature MMIC design," IEEE Transactions on Microwave Theory and Techniques, vol. 55, no. 6, pp. 1190-1199, 2007.

[12] S. Sajuyigbe, M. Ross, P. Geren, S. A. Cummer, M. H. Tanielian, and D. R. Smith, "Wide angle impedance matching metamaterials for waveguide-fed phased-array antennas," IET Microwaves, Antennas \& Propagation, vol. 4, no. 8, pp. 1063-1072, 2010.

[13] A. O. Bah, P.-Y. Qin, R. W. Ziolkowski, Y. J. Guo, and T. S. Bird, "A wideband low-profile tightly coupled antenna array with a very high figure of merit," IEEE Transactions on Antennas and Propagation, vol. 67, no. 4, pp. 2332-2343, 2019.

[14] F.-L. Jin, X. Ding, Y.-F. Cheng, B.-Z. Wang, and W. Shao, "A wideband phased array with broad scanning range and wideangle impedance matching," IEEE Transactions on Antennas and Propagation, vol. 68, no. 8, pp. 6022-6031, 2020.

[15] C.-M. Liu, S.-Q. Xiao, H.-L. Tu, and Z. Ding, "Wide-angle scanning low profile phased array antenna based on a novel magnetic dipole," IEEE Transactions on Antennas and Propagation, vol. 65, no. 3, pp. 1151-1162, 2017. 